\title{
Incorporation of Particle Swarm Optimization in Adaptive Boosting
}

\author{
Gaurav Mishra, Rohit Kumar, and Santanu Chaudhury \\ Electrical Engineering Department, Indian Institute of Technology, Delhi, India
}

\begin{abstract}
This paper proposes an optimized learning method for large feature-sets using AdaBoost to produce hardware-efficient boosted decision stumps. The paper also proposes a method for training decision stumps to construct the ensemble. AdaBoost sequentially searches for the best weak classifier in the pool and adds it to the ensemble, using weighted training samples. In the proposed method, Particle Swarm Optimization quickens the selection of decision stumps. It is shown experimentally that the optimized method is more than $60 \%$ faster than the exhaustive search method.
\end{abstract}

\section{Introduction}

An ensemble of weak classifiers is often used to produce a strong classifier 9]. AdaBoost [6, 10] provides a set of methods for combining weak classifiers to construct ensembles. Adaboost facilitates the sequential combination of weak classifiers to produce a strong ensemble, but does not address the optimal selection of these classifiers. In this paper, we propose a method that incorporates Particle Swarm Optimization [7] into AdaBoost, for the optimal selection of weak classifiers. A method to efficiently train weak decision stumps is also proposed, as these are easily implementable on hardware 4 .

Zhu et al. 2, and Zhang et al. [3] used AdaBoost to select features from histogram of oriented gradients [8], especially for human detection. Viola et al. [1] used AdaBoost to select an ensemble of decision stumps from a pool of weak stumps. In such a framework, training a large pool of stumps and their optimal selection is an important issue, which is addressed in this paper.

Evans et al. [5] used Particle Swarm Optimization (PSO) 7] to find bounds on classes with respect to all features. The image features created and used in the Viola-Jones framework are such that a gradient-descent approach can be used to find local minimas in error rates produced by using single features for training. Hence the AdaBoost procedure is suitable for and can be enhanced by PSO.

However, PSO has not been used for the design of cascaded boosted classifiers. The rest of the paper is organized in the following manner. The proposed method is described in Section 2. Experimental validation is provided in Section 3, and Section 4 concludes. 


\section{Optimized Boosting of Decision Stumps}

\subsection{Classifier Selection Using Adaboost}

An ensemble of weak classifiers can be constructed using AdaBoost, by sequentially selecting one classifier from the pool of weak classifiers and redistributing the weights of samples. In each step, the pool of classifiers is tested on the weighted training set, rated according to their performance, and the best classifier is selected. So, for large pools, exhaustive search becomes computationally expensive. AdaBoost does not address the issue of optimal selection of classifiers.

In this paper, we shall propose a method to accelerate the selection procedure of AdaBoost, using Particle Swarm Optimization. Particle Swarm optimization initializes a set of particles (classifiers from the pool, in this case) and based on their accuracy, converges towards the best weak classifier in the pool. We also propose an optimized method to create the pool of decision stumps.

\subsection{Proposed Method}

\section{Algorithm 1. AdaBoost}

1. Input $D$-dimensional training samples $S=\left(\boldsymbol{x}_{\mathbf{1}}, y_{1}\right),\left(\boldsymbol{x}_{\mathbf{2}}, y_{2}\right), \ldots\left(\boldsymbol{x}_{\boldsymbol{m}}, y_{m}\right)$ (where $\boldsymbol{x}_{\boldsymbol{i}}=\left(x_{i}^{1}, x_{i}^{2}, \ldots, x_{i}^{d}\right)^{T}$ is the $i^{\text {th }}$ sample, $y_{i}$ is the class of $i^{\text {th }}$ sample), number of iterations $P$, number of particles $N$, number of PSO iterations $T$

2. Train $D$ decision stumps $\boldsymbol{h}=\left(h_{1}, h_{2}, \ldots, h_{D}\right)$, one with respect to each feature $h_{d}: \boldsymbol{x} \mapsto\{-1,+1\}$

3. Initialize weights $w_{i}^{0}=1 / M$ for all $i=1 \ldots M$

4. Do for $p=1 \ldots P$ :

(a) Select optimal classifier $h^{p}$ using PSO, following Algorithm 2

(b) Calculate weighted error of $h^{p}: e^{p}=\sum_{i=1}^{M} w_{i}^{p} I\left(y_{i} \neq h^{p}\left(x_{i}\right)\right)$, and coefficient $\alpha^{p}=\frac{1}{2} \log \left(\frac{1-e^{p}}{e^{p}}\right)$

(c) Update the weights: $w_{i}^{p}=\frac{w_{i}^{p-1}}{Z_{p}} \exp \left\{-\alpha_{p} y_{i} h^{p}\left(x_{i}\right)\right\}$, where $Z_{p}=$ $2 \sqrt{e^{p}\left(1-e^{p}\right)}$

(d) Stop if $e^{p}=0$ or $e^{p}>1 / 2$ and set $T=t-1$

5. Output $y(x)=\operatorname{sgn}\left(\Sigma_{p=1}^{P} \alpha^{p} h^{p}(\boldsymbol{x})\right)$

\section{Algorithm 2. Optimal Selection Using PSO}

1. Input samples $S=\left\{\left(\boldsymbol{x}_{1}, y_{1}, w_{1}^{p}\right),\left(\boldsymbol{x}_{\mathbf{2}}, y_{2}, w_{2}^{p}\right), .\left(\boldsymbol{x}_{\boldsymbol{M}}, y_{M}, w_{M}^{p}\right)\right\}$, number of particles $N$, number of PSO iterations $T$, pool of decision stumps $\boldsymbol{h}=$ $\left(h_{1}, h_{2}, \ldots, h_{D}\right)$

2. Randomly select $N$ features $k^{0}=\left(k_{1}^{0}, k_{2}^{0}, \ldots, k_{N}^{0}\right)$ and corresponding decision stumps $H^{0}=\left(h_{k_{1}^{0}}, h_{k_{2}^{0}}, \ldots, h_{k_{N}^{0}}\right)$

3. Initialize velocities $V_{n}^{0}=0$ corresponding to each $h_{k_{n}^{0}}$ for $n=1 \ldots N$

4. Calculate weighted errors $e_{n}=\sum_{i=1}^{M} w_{i}^{p} I\left(y_{i} \neq h_{k_{n}^{0}}\right)$

5. Initialize global best particle $k^{*}=k_{n}^{0}:\left\{n=\operatorname{argmin}_{n} e_{n}\right\}$, and corresponding global best decision stump $h^{*}=h_{k^{*}}$ 
6. Initialize local best particles $l k_{n}^{0}=k_{n}^{0}$, local best decision stumps $l_{n}^{0}=h_{l k_{n}^{0}}$, and local best errors $l e_{n}^{0}=e_{n}^{0}$,for $n=1 \ldots N$

7. Do for $t=1 \ldots T$ :

(a) Update velocities:

$$
V_{n}^{t+1}=r_{1} V_{n}^{t}+r_{2}\left(l k_{n}^{t}-k_{n}^{t}\right)+r_{3}\left(k^{*}-k_{n}^{t}\right)
$$

(b) Update features and take corresponding decision stumps:

$$
k_{n}^{t+1}=k_{n}^{t}+V_{n}^{t} \text { for } n=1 \ldots N
$$

If $k_{n}^{t}>D$ then $k_{n}^{t}=D$, If $k_{n}^{t}<1$ then $k_{n}^{t}=1$

(c) Calculate weighted errors $e_{n}=\Sigma_{i=1}^{M} w_{i}^{p} I\left(y_{i} \neq h_{k_{n}^{t}}\left(\boldsymbol{x}_{\boldsymbol{i}}\right)\right)$

(d) Update local best stumps and errors:

$$
l k_{n}^{t}=\left\{\begin{array}{ll}
l k_{n}^{t-1} & \text { if } e_{n}^{t} \geq l e_{n}^{t} \\
k_{n}^{t} & \text { otherwise }
\end{array}, l_{n}^{t}=h_{l k_{n}^{t}} \text { and } l e_{n}^{t}=\min \left(l e_{n}^{t}, e_{n}^{t}\right)\right.
$$

(e) Update global best stump $h^{*}=\left\{l_{n}^{t}: n=\operatorname{argmin}_{n} l e_{n}^{t}\right\}$

\section{Output $h^{*}$}

In the above algorithm, $r_{1}, r_{2}$ and $r_{3}$ are the constants used to control the movement of the particles. Empirical studies have recommended the values $r_{1}=$ $0.729844, r_{2}=r_{3}=1.4961798$ for the optimal convergence of PSO [1]. Van den Berg 12 has shown that PSO is locally convergent. Hence, if enough particles are initailized over the search space, the probability of reaching the global minima is high. Empirical analysis has shown PSO to be reliable optimization technique[7.

\subsection{Training Decision Stumps}

To train decision stumps described in the proposed method, a similar approach using PSO can be used. For example, consider a $256 \times 2562$-D sample space. Suppose we select only integer stumps, then a threshold has to be selected from 256 possibilities w.r.t. one feature. Instead of an exhaustive search, an optimized search using PSO can be executed.

\section{Experimental Validation}

\subsection{Datasets Used}

The proposed method was tested on 3 databases, the INRIA pedestrian databas 1 , a database of faces 2 , and the Gissette databas! 3 (see Table1). The INRIA dataset

\footnotetext{
${ }^{1}$ Available at www pascal.inrialpes.fr/data/human/

${ }^{2}$ Available at cswww.essex.ac.uk/mv/allfaces/index.html

${ }^{3}$ Available in the UCI repository 13 , www.archive.ics.uci.edu/ml/datasets/Gisette
} 
provides images of pedestrians. The Face database contains images of faces to use for training. HOG features were extracted from these images to train the decision stumps. For the other datasets, extracted features were available and were used for training purposes. Decision stumps were trained in the following manner: for each feature, the mean value of both classes was taken, and the mean of these two values was taken as the threshold.

Table 1. Description of Datasets

\begin{tabular}{|l|c|c|}
\hline Dataset & Number of Samples & Number of features \\
\hline INRIA & 3634 & 1610 \\
Faces & 6078 & 4140 \\
Gisette & 6000 & 5000 \\
\hline
\end{tabular}

The time required for training and the accuracy of resulting ensembles using exhaustive search and optimized search were compared using these feature sets.

\subsection{Results}

The overall error as more features are added to the ensemble is plotted w.r.t. training time in Fig. 1,2, and 3. The overall classification error decreases exponentially as more weak classifiers are added [6]. Clearly, the optimized search takes less time construct an ensemble than exhaustive search. The training time taken to achieve $10 \%$ error rate, $5 \%, 2 \%$ and zero classification error are tabulated in Table 2. The improvement in training times using PSO search are tabulated in Table 3 . In most cases, an improvement of more than $60 \%$ is observed.

The training method for decision stumps using PSO was tested using a sample $2 \mathrm{D}$ dataset shown in Fig. 4. An ensemble of 50 decision stumps is shown in Fig. 5 and of 200 decision stumps is shown in Fig. 6. Using more stumps gives a closer approximation of the linear boundary between the classes.

Table 2. Training Time(sec)

\begin{tabular}{|c|c|c|c|c|c|c|c|c|}
\hline \multirow[t]{2}{*}{ Dataset } & \multicolumn{2}{|c|}{$10 \%$ error } & \multicolumn{2}{|c|}{$5 \%$ error } & \multicolumn{2}{|c|}{$2 \%$ error } & \multicolumn{2}{|l|}{0 error } \\
\hline & Exhaustive & $\mathrm{PSO}$ & Exhaustive & $\overline{\mathrm{PSO}}$ & Exhaustive & $\mathrm{PSO}$ & Exhaustive & $\mathrm{PSO}$ \\
\hline INRIA & 4 & 1 & 13 & 6 & $\overline{49}$ & 21 & 274 & 106 \\
\hline Face & 19.7 & 3.8 & 45.6 & 15.2 & 175.4 & 60.9 & 1501.8 & 425.7 \\
\hline Gisette & 21 & 8 & 108 & 41 & 506 & 198 & 1970 & 777 \\
\hline Car & 5.6 & 0.8 & 20.8 & 8.4 & - & - & - & - \\
\hline
\end{tabular}




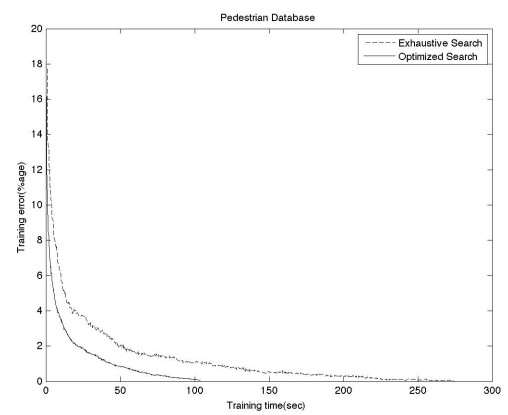

Fig. 1. Pedestrian: Training Error(\%age) vs. Training Time(sec)

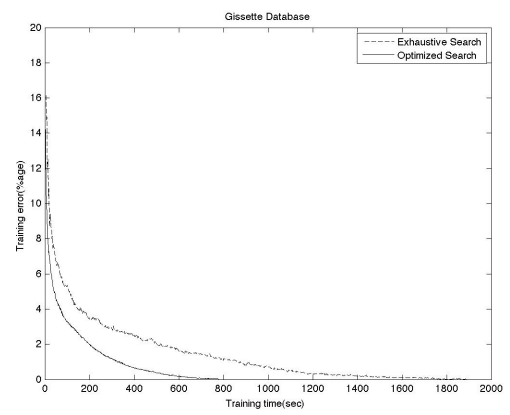

Fig. 3. Gissette: Training Error(\%age) vs. Training Time(sec)

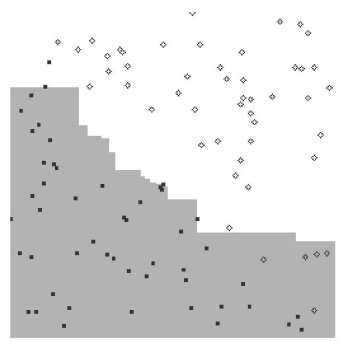

Fig. 5. Training Decision Stumps: 50 stumps

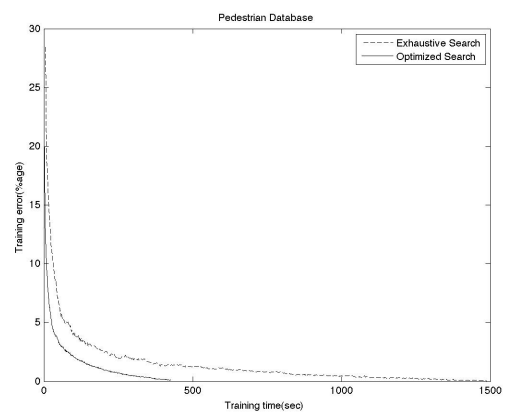

Fig. 2. Faces: Training Error(\%age) vs. Training Time(sec)

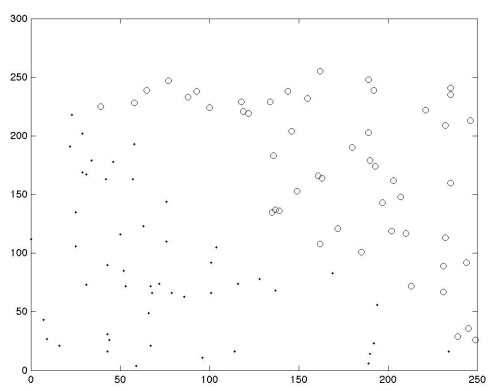

Fig. 4. Sample 2D Dataset

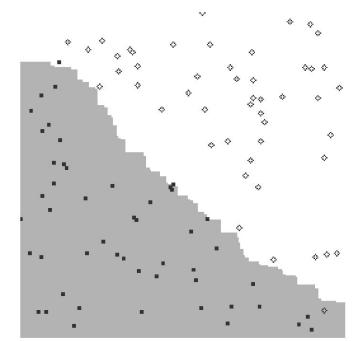

Fig. 6. Training Decision Stumps: 200 stumps 
Table 3. Improvement in Training Time(\%age)

\begin{tabular}{|l|c|c|c|c|}
\hline Dataset & 10\%error & $5 \%$ error & $2 \%$ error & 0 error \\
\hline INRIA & 75 & 53.84 & 57.14 & 61.31 \\
Face & 80.71 & 66.67 & 65.27 & 71.65 \\
Gisette & 61.9 & 62.03 & 60.86 & 60.55 \\
Car & 85.71 & 59.61 & - & - \\
\hline
\end{tabular}

\section{Conclusion}

In this paper we presented a modified approach to Adaptive Boosting for object detection applications. Particle Swarm Optimization was used to improve the time taken to construct ensembles. It was demonstrated that this reduction was more than $60 \%$. This method can be used to produce ensembles in a faster and more efficient manner.

\section{References}

[1] Viola, P., Jones, M.: Robust Real-time Object Detection. International Journal of Computer Vision (2001)

[2] Zhu, Q., Avidan, S., Yeh, M., Cheng, K.: Fast human detection using a cascade of histograms of oriented gradients. In: CVPR 2006, pp. 1491-1498 (2006)

[3] Jia, H., Zhang, Y.: Fast Human Detection by Boosting Histograms of Oriented Gradients. In: Fourth International Conference on Image and Graphics, pp. 683-688 (2007)

[4] Miteran, J., Matas, J., Bourennane, E., Paindavoine, M., Dubois, J.: Automatic hardware implementation tool for a discrete adaboost-based decision algorithm. EURASIP Journal on Applied Signal Processing, 1035-1046 (2005)

[5] Evans, H., Zhang, M.: Particle swarm optimisation for object classification. In: 23rd International Conference on Image and Vision Computing, New Zealand, pp. 1-6 (2008)

[6] Freund, Y., Schapire, R.E.: A Decision-Theoretic Generalization of on-Line Learning and An Application to Boosting (1995)

[7] Kennedy, J., Eberhart, R.: Particle swarm optimization Proceedings. In: IEEE International Conference on Neural Networks, vol. 4, pp. 1942-1948 (1995)

[8] Dalal, N., Triggs, B.: Histograms of Oriented Gradients for Human Detection. In: CVPR, pp. 886-893 (2005)

[9] Kuncheva, L.I.: Combining Pattern Classifiers: Methods and Algorithms. WileyInterscience (2004)

[10] Schapire, R.E., Singer, Y.: Improved boosting algorithms using confidence-rated predictions. In: Proceedings of the 11th Annual Conference on Computational Learning Theory, pp. 80-91. ACM (1998)

[11] Eberhart, R.C., Shi, Y.: Comparing Inertia Weights and Constriction Factors in Particle Swarm Optimization. In: Proceedings of the IEEE Congress on Evolutionary Computation, San Diego, USA (2000)

[12] Bergh, F., Engelbrecht, A.P.: A Convergence Proof for the Particle Swarm Optimiser (2010)

[13] Guyon, I., Gunn, S.R., Ben-Hur, A., Dror, G.: Result analysis of the NIPS 2003 feature selection challenge (2004) 